\title{
Wandering Kidney in Dual-Kidney Transplant Recipient Presents a Pitfall in the Interpretation of Renal Scintigraphy
}

\author{
Nathan D. Anavy, Chirag S. Desai, Ryan Avery, and Phillip H. Kuo \\ Department of Medical Imaging, University of Arizona, Tucson, Arizona
}

\begin{abstract}
A 26-y-old patient with end-stage renal disease and recent dual transplantation of cadaveric kidneys en bloc presented with increasing abdominal pain and a rising level of serum creatinine. An anterior-view ${ }^{99 m}$ Tc-mercaptoacetyltriglycine renogram demonstrated the typical overlap of the lower pole of the superior kidney and the upper pole of the inferior kidney. The renogram was consistent with vasomotor nephropathy. Subsequent imaging $1 \mathrm{wk}$ later for worsening symptoms demonstrated a single reniform structure in the expected location of the inferior transplanted kidney, which was interpreted as a loss of perfusion to the superior kidney. Correlation with subsequent CT and sonography showed normal perfusion to both transplanted kidneys and that the superior kidney had wandered inferiorly, completely overlapping the inferior kidney on the anterior projection. The increasing prevalence of dual kidney transplantation warrants special attention to the potential for a wandering kidney.
\end{abstract}

Key Words: dual renal transplant; MAG-3; wandering kidney

J Nucl Med Technol 2012; 40:275-277

DOI: 10.2967/jnmt.111.101592

$\mathbf{T}$ he use of dual-kidney transplants from pediatric or marginal adult donors has increased as techniques to maximize the availability of potential donor organs have evolved $(1,2)$. Various surgical techniques for such transplantations have been developed, including en bloc and split individual implantation (3). Renal scintigraphy is an invaluable tool for the assessment of renal transplants because of its safety and its ability to assess perfusion, function, clearance, and urine leakage $(4,5)$. However, dual en bloc transplantations may present some pitfalls in the interpretation of renal scintigraphy. Here, we describe a dual-transplantation patient in whom one of the transplanted kidneys "wandered," overlapping

\footnotetext{
Received Dec. 9, 2011; revision accepted Apr. 2, 2012.

For correspondence or reprints contact: Nathan Anavy, University of Arizona Medical Center, Department of Medical Imaging, 1501 N. Campbell Ave., Tucson, AZ 85724.

E-mail: nate.anavy@gmail.com

Published online Sep. 20, 2012.

COPYRIGHT (C 2012 by the Society of Nuclear Medicine and Molecular Imaging, Inc.
}

the other kidney and resulting in an inaccurate renography interpretation.

\section{CASE REPORT}

A 26-y-old patient with end-stage renal disease and recent dual transplantation of pediatric kidneys en bloc presented for ${ }^{99 \mathrm{~m} T c-m e r c a p t o a c e t y l t r i g l y c i n e ~(99 m T c-M A G 3) ~}$ renography because of increasing abdominal pain and a rising level of serum creatinine. The initial renogram for this patient was obtained $3 \mathrm{~d}$ after transplantation. The anterior view demonstrated overlap of the lower pole of the superior kidney and the upper pole of the inferior kidney in the right pelvis (Fig. 1). Arterial and delayed images showed satisfactory perfusion and uptake to both transplanted kidneys.

The renography results were consistent with vasomotor nephropathy. Vasomotor nephropathy, previously but still often known as posttransplantation acute tubular necrosis, occurs when blood flow is inadequate to maintain renal function yet the inadequacy is not so complete or sustained that infarction results. The condition is reversible. On renography the entity manifests as decreased renal function with decreased overall radiotracer uptake and delayed cortical clearance (6). These findings suggest but are not specific for vasomotor nephropathy and may require biopsy with pathology being the gold standard of diagnosis.

On postoperative day 10, the patient presented with increasing abdominal pain and a worsening serum creatinine level. Repeated scanning (Fig. 2) demonstrated a single reniform structure in the expected location of the more inferiorly located transplanted kidney as seen on the prior renogram. The standard protocol at our institution includes obtaining a lateral projection after administration of furosemide (Lasix; Sanofi-Aventis); however, the patient was not able to complete the study because of severe pain.

The lack of visualization of a second reniform structure suggested loss of perfusion to a kidney. The most emergent differential considerations for vascular occlusion of a renal transplant include surgical complications or organ rejection, both of which can have significant morbidity. Surgical complications include stenosis at the anastomosis, dehiscence, or mechanical obstruction, with higher rates expected with the more complicated dual en bloc procedure (7). Organ rejection may be hyperacute or acute. Hyperacute rejection occurs almost immediately on reperfusion 
FIGURE 1. Anterior-view 99mTc-MAG3 renogram obtained $3 \mathrm{~d}$ after transplantation. Lower pole of superior kidney and upper pole of inferior kidney overlap. Expected radiotracer accumulation in region of urinary bladder is not seen (arrowhead).

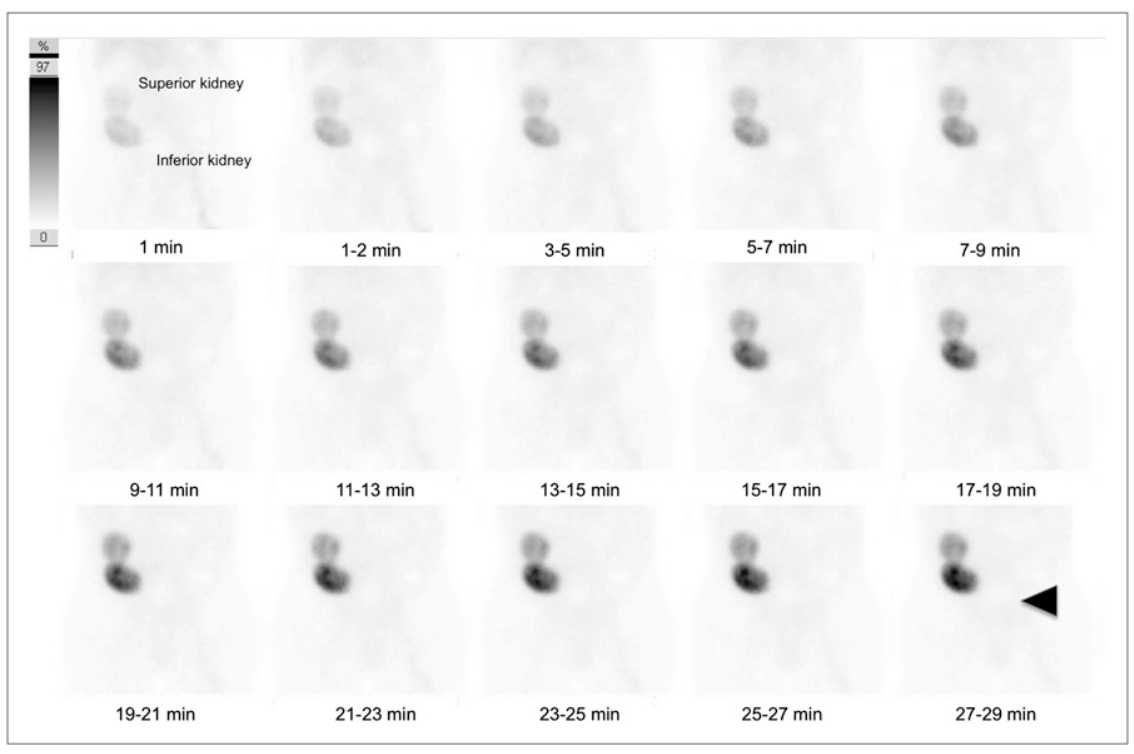

of the donor kidney within the recipient with activation of preformed antibodies to the donor organ. Acute rejection results from activation of the immune system with peak incidence occurring 1-3 wk after transplantation (6). Both types of rejection ultimately result in vascular endothelial damage and thrombosis. The time frame of this case favored acute rejection. Total lack of arterial flow and then lack of visualization of uptake on delayed imaging favor arterial over venous failure, but both arterial and venous complications should be considered in transplant evaluation.

The nuclear physician immediately notified the surgical team of the suspicion of vascular occlusion and recommended emergent sonography to evaluate perfusion. Doppler sonography showed adequate flow to both kidneys and a change in the position of the kidneys relative to each other with new alignment in the anteroposterior plane (Fig. 3). Because the patient's severe abdominal pain led to concerns about ischemic bowel, a CT scan with intravenous iodinated contrast material was also performed. The scan clearly demonstrated the nearly complete overlap of the kidneys in the anteroposterior dimension (Fig. 4). The contrast material further confirmed perfusion of both kidneys. Three days later, another ${ }^{99 \mathrm{~m}}$ Tc-MAG3 scan was performed (Fig. 5), and this time, lateral views were obtained. Lateral planar images before and after furosemide administration confirmed that the 2 transplanted kidneys were aligned anteroposteriorly as seen on sonography and CT.

The ultimate cause of the patient's pain was a rare case of necrotic ureter with an otherwise healthy transplanted kidney. The transplant surgeon removed both the necrotic ureter
FIGURE 2. Anterior-view 99mTc-MAG3 renogram obtained $7 \mathrm{~d}$ after initial renogram. Single reniform shape is seen (arrow), suggesting loss of perfusion and function in superior renal transplant. Radiotracer is seen within bladder (arrowhead).

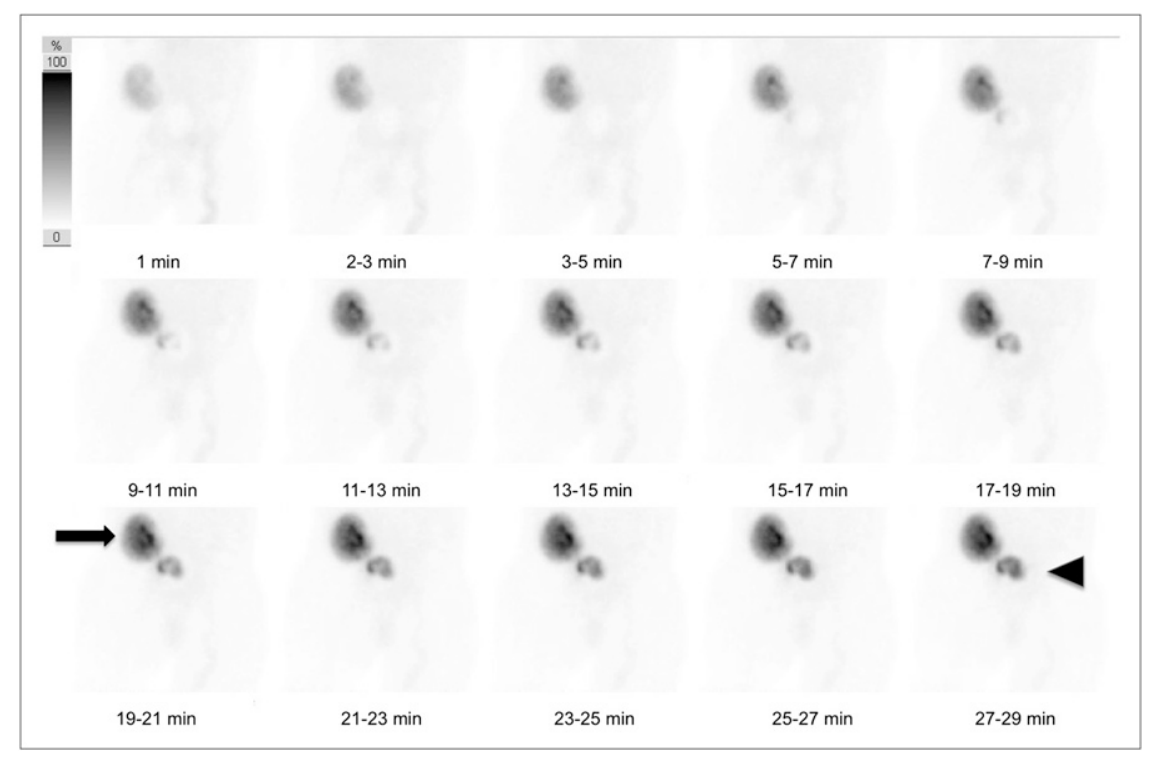




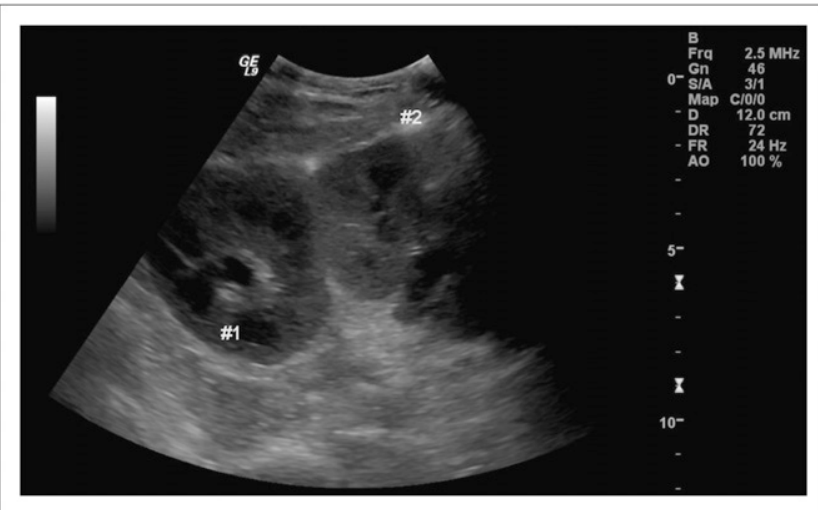

FIGURE 3. Sonogram of transplanted kidneys, demonstrating anatomic overlap.

and its corresponding kidney, and the pain subsequently resolved. The patient was eventually discharged from the hospital but required hemodialysis because the remaining pediatric kidney transplant provided insufficient filtration.

\section{DISCUSSION}

The increasing use of dual-kidney transplantation warrants that nuclear physicians pay special attention to the potential for shifting anatomy during renal scintigraphy. In this case, a wandering transplanted kidney nearly resulted in unnecessary emergent surgery. If additional imaging had not been performed, the patient would have been reexplored for vascular occlusion of the transplanted kidney. This case resulted in a change in the standard protocol at our institution. The transplant surgeons at our institution typically request renal scintigraphy with furosemide. Previously, only at the end of the examination did the technologists obtain a lateral view to evaluate for urine leakage. Now, the technologists obtain a lateral view immediately after the prefurosemide imaging and also at the end of the postfurosemide imaging in case imaging is terminated early.

The lateral view will generally give the interpreter enough information to clarify the shifting anatomy. SPECT/CT is

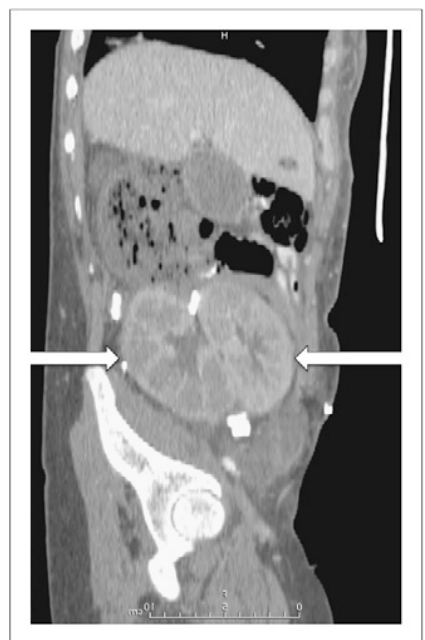

FIGURE 4. Sagittal reconstruction of abdominal and pelvic CT scan. Essentially complete overlap of transplanted kidneys (arrows) in anteroposterior plane is seen to be cause of renography misinterpretation.

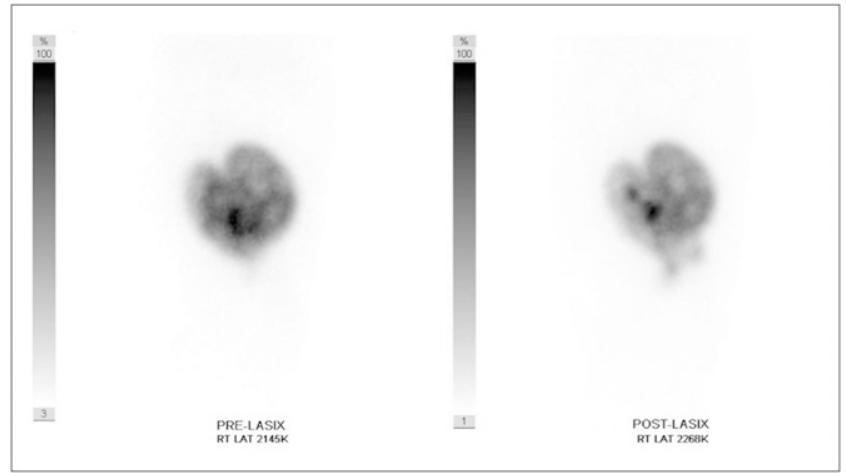

FIGURE 5. Three days after CT, planar lateral-view 99mTcMAG3 scans obtained before and after furosemide confirm that the 2 transplanted kidneys are aligned anteroposteriorly as seen on sonography and CT.

a consideration and would have been helpful for anatomic correlation, for clarification of unusual or altered anatomy, and for prevention of the subsequent abdominal CT the patient underwent. In this case, since the patient could no longer tolerate nuclear imaging, Doppler sonography was used to assess anatomy and blood flow. Sonographic confirmation should be considered after nuclear renography in situations such as a suboptimal flow study from a poor bolus or attenuation.

\section{CONCLUSION}

Misinterpretation of anterior-projection renal scintigraphy findings can occur if dual renal transplants shift and completely overlap each other. Due diligence for this pitfall is essential to prevent misdiagnosis of transplant failure and possible unnecessary surgery.

\section{ACKNOWLEDGMENT}

No potential conflict of interest relevant to this article was reported.

\section{REFERENCES}

1. Laftavi MR, Stephan R, Feng L, et al. Dual kidney transplants from very old or very young donors: long-term outcomes and complications. Transplant Proc. 2011;43:507-511.

2. Bunnapradist S, Gritsch HA, Peng A, et al. Dual kidneys from marginal adult donors as a source for cadaveric renal transplantation in the United States. $J A m$ Soc Nephrol. 2003;14:1031-1036.

3. Johnson LB, Kuo PC, Dafoe DC, et al. Double adult renal allografts: a technique for expansion of the cadaveric kidney donor pool. Surgery. 1996;120: 580-583.

4. Brown ED, Chen MY, Wolfman NT, et al. Complications of renal transplantation: evaluation with US and radionuclide imaging. Radiographics. 2000;20:607-622.

5. American College of Radiology Web site. ACR-SPR practice guideline for the performance of adult and pediatric renal scintigraphy. Available at: http://www. acr.org/ /media/1169D04DFABF4C10938D2E3DFADC4477.pdf. Revised 2008. Accessed September 7, 2012.

6. Park SB, Kim JK, Cho KS. Complications of renal transplant: ultrasonographic evaluation. J Ultrasound Med. 2007;26:615-633.

7. Bhayana S, Kuo YF, Madan P, et al. Pediatric en bloc kidney transplantation to adult recipients: more than suboptimal? Transplantation. 2010;90:248-254. 